\title{
ANÁLISE DO COMPORTAMENTO MECÂNICO DE MACIÇOS ROCHOSOS CONTENDO DESCONTINUIDADES ATRAVÉS DO MÉTODO DAS DIFERENÇAS FINITAS VIA IMPLEMENTAÇÃO COMPUTACIONAL
}

\author{
Analysis of the mechanical behavior of rocky mass with discontinuities \\ through the finite differences method via computational implementation
}

Luma Alvarenga Carvalho de Vasconcelos ${ }^{1}$, Pedro Guilherme Cipriano Silva ${ }^{2}$, Pedro Ivo Amaro Alves ${ }^{3}$

Recebido em 18 de janeiro de 2018; aceito em 27 de maio de 2018; disponível on-line em 28 de agosto de 2018.

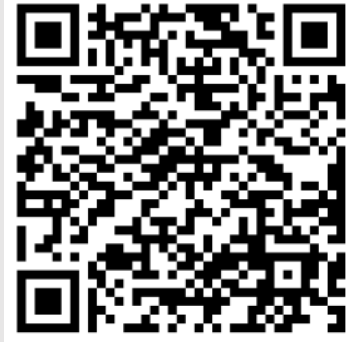

PALAVRAS CHAVE:

Maciço rochoso;

Diferenças finitas;

Teoria da elasticidade;

Descontinuidade.

\section{KEYWORDS:}

Rocky mass;

Finite differences;

Theory of elasticity;

Discontinuity.

\footnotetext{
* Contato com os autores:
}

${ }^{1}$ e-mail: luma-alvarenga@hotmail.com ( L. A. C. de Vasconcelos )

Engenheira de Minas, Mestre em Geotecnia, Doutoranda em Engenharia Mineral, Universidade Federal de Ouro Preto.

2e-mail: pedrogcs@hotmail.com (P.G.C. Silva )

Engenheiro de Minas, Mestrando em Engenharia Mineral, Universidade Federal de Ouro Preto.

${ }^{3}$ e-mail: pedroivominas@gmail.com ( P. I. A. Alves )

Engenheiro de Minas, Mestrando em Engenharia Mineral, Universidade Federal de Ouro Preto.
ABSTRACT: In this work a computational analysis is performed by solving differential equations for the modeling of numerical (MDF) and analytical solutions of the mechanical behavior of a rock mass with discontinuities. Simulations were carried out by adopting a composed of a homogeneous, isotropic and linear elastic material under the action (t) compressive force applied at its free end and confined between two rigid discontinuities on the lower and upper surfaces. The lower discontinuity presents roughness characterized by a shear stiffness modulus leading to a shear stress distribution and a relative displacement between the block and the base. The results were ted through the compilation of the data in the FORTRAN 90 computer program, and with this, it is possible to verify the relative displacement between the block and the discontinuity, as well as the shear stresses acting at each previously defined point; and also perform a comparison between analytical and numerical solutions. The main tribution of the work to the area of geotechnics is the possibility of an analysis of discontinuity displacement present in rock mass closer to reality since it considers the environment as discontinuous. In addition, a computational solution is presented that is easy to implement and understand. 


\section{INTRODUÇÃO}

O estudo do comportamento mecânico de maciços rochosos contendo descontinuidades é fundamental na análise de estabilidade de taludes e de escavações subterrâneas. A maioria das rupturas de taludes a céu aberto, colapsos de pilares e queda de blocos em minerações subterrâneas ocorre ao longo de planos de fraqueza em um movimento cisalhante. As propriedades da rocha intacta e das descontinuidades são as principais condicionantes desse comportamento.

Relacionado as descontinuidades, em geral, os projetos de análise de estabilidade requerem a modelagem correta das propriedades de deformabilidade, resistência e condutividade que são as responsáveis por condicionar o comportamento do maciço rochoso em ambientes de baixas tensões. Segundo Vargas Jr. (1991) as descontinuidades representam a parte fraca da mecânica dos maciços rochosos, possuem alta deformabilidade, baixa resistência ao cisalhamento e à tração e são as responsáveis por estabelecerem os caminhos preferenciais de percolação de fluidos, caso existam.

De maneira geral as soluções - tanto numéricas, quanto analíticas - que envolvem as análises de estabilidade de taludes e escavações subterrâneas são feitas considerando-se os maciços rochosos como meios contínuos utilizando parâmetros geotécnicos equivalentes para adaptar aos problemas descontínuos. Entretanto, essa metodologia não é suficiente para reproduzir o comportamento mecânico real dos maciços rochosos contendo descontinuidades.

Nos últimos anos estudos e pesquisas vem sendo desenvolvidas com a finalidade de criar metodologias que possibilitem a obtenção de resultados mais próximos da realidade possível. Diante desse cenário o presente trabalho possui como objetivo realizar uma análise numérica via MDF com implementação computacional para avaliar o comportamento mecânico em termos de deslocamento relativo e tensão cisalhante de um maciço rochoso confinado entre duas descontinuidades considerando-se um modelo constitutivo elástico perfeitamente plástico.

\section{CONTEXTUALIZAÇÃO}

A rocha é um material geológico natural formado por diferentes minerais. É descontínuo, anisotrópico, não homogêneo, inelástico e contém várias zonas de potenciais falhas. Devido à essa natureza irregular, a previsibilidade de seu comportamento mecânico sempre foi um desafio para a engenharia, uma vez que este conhecimento é necessário para o dimensionamento de projetos que levam à utilização de rochas: fundações, escavações subterrâneas, contenções de teto em minas subterrâneas, construções de estradas, casas e prédios, estabilidade de encostas, entre outros.

Para a avaliação do comportamento mecânico de um maciço rochoso contendo descontinuidades é comum realizar a análise em termos de deslocamento relativo e tensão cisalhante entre o maciço e a descontinuidade considerando as descontinuidades como interfaces.

Muitos modelos foram e vem sendo publicados para avaliar o comportamento mecânico de maciços rochosos descontínuos sob atuação de tensões cisalhantes tais como: Patton (1966), Goodman (1968), Ladanyi e Archambault (1969), Bandis et al. (1983), Plesha (1988), Saeb e Amadei (1992), Seidel e Haberfield (2002a, b) e Grasselli (2006).

A modelagem matemática e classificações geomecânicas são importantes ferramentas utilizadas em mecânica de rochas. Contudo, o avanço tecnológico computacional permitiu a utilização de métodos numéricos para resolução dos modelos matemáticos complexos originados no estudo destes materiais e são usados hoje como a principal abordagem em design de engenharia e pesquisa de mecânica de rochas.

Dentro deste campo, Nikolić (2016) aborda a utilização dos métodos numéricos 
existentes, percorrendo didaticamente sobre o funcionamento de cada um, e destaca o método das diferenças finitas, como o método mais direto e intuitivo, além de ser amplamente utilizado devido à sua simplicidade, pois não utiliza funções de interpolação como em outros métodos e possui como vantagem a possibilidade de simulação de comportamento de material não-linear complexo sem soluções iterativas.

O presente trabalho utiliza o modelo constitutivo proposto por Goodman (1968) para avaliar a tensão cisalhante e deslocamento relativo entre a interface e o maciço rochoso através do método das diferenças finitas com implementação computacional.

A escolha do modelo constitutivo se baseou nos trabalhos de Hird and Russel (1990) que propuseram uma solução analítica e em método dos elementos finitos baseada no modelo constitutivo em questão, e foi utilizada neste trabalho para fins comparativos. Ainda, Pereira (2003) utilizou o modelo proposto pelo autor e propôs um elemento de interface na análise por método dos elementos finitos considerando a interface entre solo e elemento de reforço (geossintéticos) e boa representatividade da realidade foi observada nos resultados. Usefzadeh (2013) também propôs um modelo empírico baseado no modelo de Goodman e em resultados de ensaios laboratórios.

O modelo de Goodman (1968) leva em consideração os conceitos de rigidez normal $\left(k_{n}\right)$ para descrever a taxa de modificação da tensão normal em relação aos deslocamentos normais e rigidez cisalhante $\left(k_{s}\right)$ para descrever a taxa de modificação da tensão cisalhante em relação aos deslocamentos cisalhantes.

Assim, considera-se o problema como elástico perfeitamente plástico, analisando apenas os movimentos derivados da tensão cisalhante, considerando um maciço rochoso contendo uma descontinuidade cuja rugosidade seja caracterizada pelo valor de $\mathrm{k}_{\mathrm{s}}$. Caso o maciço esteja sob atuação de uma força compressiva, uma tensão cisalhante será induzida na interface entre
- maciço e a descontinuidade e consequentemente surgirá um deslocamento cisalhante na mesma até que a tensão atinja a resistência ao cisalhamento do material $\tau_{\text {máx }}$ equivalente a um valor de deslocamento relativo cisalhante $w_{1}$, conforme indicado no gráfico da Figura 1.

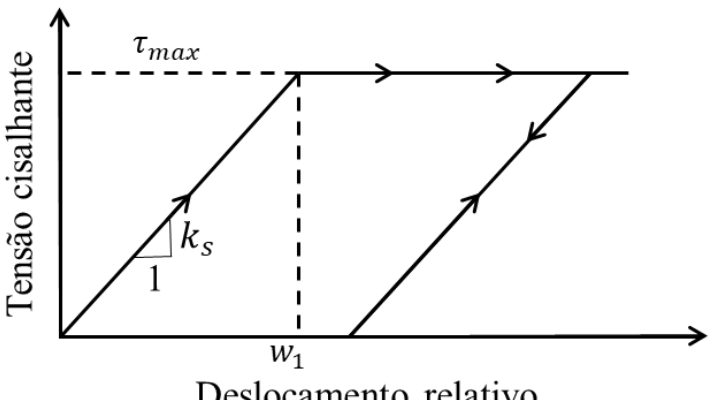

FIGURA 1: Comportamento constitutivo de uma descontinuidade com superfície rugosa. FONTE: Adaptado de Hird \& Hussel (1990).

\section{METODOLOGIA}

Para o desenvolvimento da solução numérica com posterior implementação computacional do problema apresentado nesse artigo, primeiramente foram realizados 0 conhecimento e apresentação do modelo físico. Então, o modelo matemático pôde ser elaborado adotando-se o modelo proposto por Goodman (1968) e com base no trabalho de Hird e Russell (1990) que também apresentaram uma solução analítica para o problema, utilizada neste trabalho para fins comparativos. Sequencialmente a formulação numérica via método das diferenças finitas foi proposta e implementada computacionalmente. Por fim, um modelo exemplar foi avaliado para comparação das soluções analítica e numérica e validação da implementação computacional.

\subsection{APRESENTAÇÃO DO MODELO FíSICO}

Para simular a situação analisada considera-se um problema mecânico de equilíbrio estático. O maciço rochoso é representado por um bloco de altura $\mathrm{H}$ e comprimento $\mathrm{L}$ composto por um material homogêneo, isotrópico e linear 
elástico e sob atuação de uma força externa compressiva aplicada na sua extremidade livre $(x=L)$. As descontinuidades são representadas por duas superfícies (inferior e superior) que são responsáveis pelo confinamento do maciço. A descontinuidade inferior apresenta rugosidade, caracterizada por um módulo de rigidez ao cisalhamento $\left(k_{s}\right)$ que conduz a uma distribuição de tensão cisalhante e a um deslocamento relativo $(w(x))$ entre o bloco e a base, cujo comportamento será avaliado. A descontinuidade superior é rígida e lisa, conduzindo a uma distribuição de tensão de cisalhamento nula ao longo da interface superior do bloco. O modelo físico considerado está representado na Figura 2.

\subsection{ELABORAÇÃO DO MODELO MATEMÁTICO}

Para a elaboração do modelo matemático utilizou-se um elemento infinitesimal de altura $\mathrm{H}$ e comprimento $\mathrm{dx}$ pertencente ao modelo físico do problema, conforme representado na Figura 3.

O elemento está sob atuação da tensão normal $\sigma_{\mathrm{x}}$ definida como a tensão atuante na extremidade não fixa do bloco devido a carga $P$ aplicada horizontalmente, e existe ainda a tensão cisalhante $\tau$ na interface bloco-descontinuidade originada devido a rugosidade da superfície e do seu módulo de rigidez ao cisalhamento ks.

Aplicando-se o equilíbrio estático ao elemento infinitesimal adotado obtém-se a Equação 1:

$$
\tau d x-H \frac{d \sigma_{x}}{d x} d x=0
$$

Em que:

$\boldsymbol{\tau}=$ tensão de cisalhamento $\left(\mathrm{kN} / \mathrm{m}^{2}\right)$;

$\mathbf{d x}=$ comprimento do elemento infinitesimal $(\mathrm{m})$;

$\mathbf{H}=$ altura do elemento infinitesimal (m);

$\sigma_{\mathbf{x}}=$ tensão normal horizontal ao elemento infinitesimal $\left(\mathrm{kN} / \mathrm{m}^{2}\right)$;

$\mathbf{d} \sigma_{\mathbf{x}}=$ variação da tensão normal horizontal ao longo de $d x\left(k N / m^{2}\right)$.

De acordo com a teoria da elasticidade, a tensão de cisalhamento está relacionada ao deslocamento longitudinal através do módulo de rigidez cisalhante $k_{s}$ segundo a Equação 2.

$$
\tau(x)=k_{s} \cdot w(x)
$$

Em que:

$\boldsymbol{\tau}(\mathbf{x})=$ tensão de cisalhamento ao longo da posição $\mathrm{x}\left(\mathrm{kN} / \mathrm{m}^{2}\right)$;

$\mathbf{k}_{\mathrm{s}}=$ módulo rigidez cisalhante $(\mathrm{kN} / \mathrm{m})$;

$\mathbf{w}(\mathbf{x})$ = deslocamento relativo ao longo da posição $x(m)$.

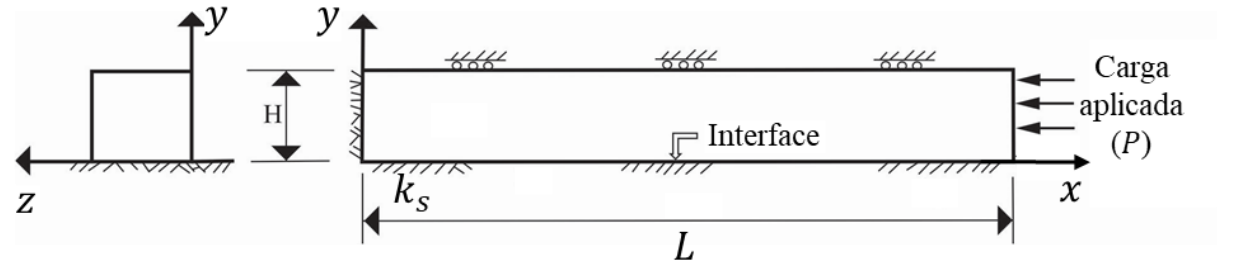

FIGURA 2: Representação do modelo físico adotado.

FONTE: Autoria própria.

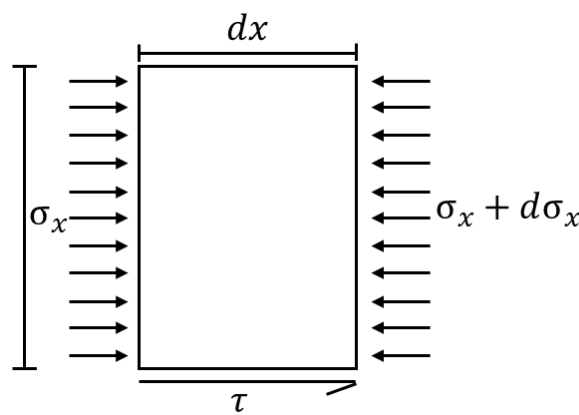

FIGURA 3: Elemento infinitesimal adotado na elaboração do modelo matemático. FONTE: Autoria própria. 
Ainda, de acordo com a teoria da elasticidade a tensão normal na direção longitudinal do bloco $\sigma_{\mathrm{x}}$ pode ser escrita, considerando uma condição de deformação plana, conforme Equação 3:

$$
\sigma_{\mathrm{x}}=\frac{1}{\mathrm{~m}_{\mathrm{v}}} \varepsilon_{\mathrm{x}}
$$

Em que:

$\varepsilon_{\mathrm{x}}=$ deformação longitudinal do bloco $(\mathrm{m})$, obtida através da Equação 4.

$$
\varepsilon_{\mathrm{x}}=-\frac{\mathrm{dw}}{\mathrm{dx}}
$$

$\mathbf{d} \mathbf{w}$ = variação do deslocamento relativo;

$\mathbf{d x}=$ variação da posição;

$\mathbf{m}_{\mathbf{v}}=$ módulo de deformabilidade, obtido através da Equação 5.

$$
m_{v}=\frac{(1+v)(1-2 v)}{E(1-v)}
$$

$E=$ Módulo de Young $\left(\mathrm{kN} / \mathrm{m}^{2}\right)$;

$\mathbf{v}=$ Coeficiente de Poisson.

Substituindo-se a Equação 5 na Equação 3, e substituindo o resultado junto com a Equação 2 na Equação 1, chega-se à seguinte equação diferencial ordinária homogênea (Equação 6):

$$
\frac{d^{2} w}{d x^{2}}+\alpha^{2} w=0
$$

Em que:

$$
\boldsymbol{\alpha}=\sqrt{\frac{k_{s} m_{v}}{H}}
$$

A equação diferencial ordinária homogênea, Equação 6, é válida para todo comprimento do bloco. No entanto, ela deve atender à condição de contorno natural na extremidade livre ( $x=\mathrm{L}$ ) (Equação 7):

$$
\frac{d w}{d x}=-m_{v} H P
$$

e atender à condição de contorno essencial em $x=0$, em que $w(x=0)=0$.

É importante enfatizar que a Equação 6 só é válida para valores de carregamento externo $\mathrm{P}$ para o qual a tensão de cisalhamento na base do bloco é no máximo igual à resistência ao cisalhamento na interface bloco-descontinuidade.

\subsection{SOLUÇÃO ANALÍTICA}

Para a determinação da solução analítica a equação diferencial exibida na Equação 6 foi resolvida. Nesse processo considerou-se a relação entre a tensão cisalhante e o deslocamento relativo como elástica perfeitamente plástica e adotou-se o critério de ruptura de Mohr-Coulomb, definindo-se a tensão cisalhante máxima em função da coesão como representado na Equação 8 e consequentemente definiu-se também o valor do deslocamento relativo ao atingir-se esse valor definido pela Equação 9:

$$
\begin{aligned}
\tau_{\max } & =\mathrm{c} \\
\mathrm{w}_{1} & =\frac{\mathrm{c}}{\mathrm{k}_{\mathrm{s}}}
\end{aligned}
$$

Em que:

$\boldsymbol{\tau}_{\text {máx }}=$ resistência ao cisalhamento $\left(\mathrm{kN} / \mathrm{m}^{2}\right)$;

c = coesão $\left(\mathrm{kN} / \mathrm{m}^{2}\right)$;

$\mathbf{w}_{\mathbf{1}}=$ deslocamento relativo ao se atingir a resistência ao cisalhamento $(\mathrm{m})$.

A partir da resolução da Equação 6 com substituição das Equações 8 e 9 chegou-se a Equação 10, também proposta por Hird and Russel (1990).

$$
\left(\frac{\mathrm{e}^{\alpha \mathrm{x}_{1}}-\mathrm{e}^{-\alpha x_{1}}}{\mathrm{e}^{\alpha \mathrm{x}_{1}}+\mathrm{e}^{-\alpha \mathrm{x}_{1}}}\right)+\alpha\left(\mathrm{L}-\mathrm{x}_{1}\right)-\frac{\mathrm{pH \alpha}}{\tau_{\max }}=0 \quad \text { Eq.[10] }
$$

\section{Em que:}

$\mathbf{x}_{\mathbf{1}}=$ posição a partir da qual a tensão atinge a tensão cisalhante máxima (m).

$O$ valor de $\mathrm{x}_{1}$ pode ser obtido a partir da resolução da Equação 10. Para a situação em que a tensão cisalhante máxima ainda não tenha sido atingida as equações que regem o comportamento mecânico do maciço rochoso em termos de deslocamento relativo e tensão cisalhante podem ser obtidas aplicando-se à E.D.O representada na Equação 6 as condições de contorno essenciais em que para $x=0, w(x=0)=0.0$ e para $x=x_{1}, w\left(x=x_{1}\right)=w_{1}$, chegando-se às Equações 11 e 12. 


$$
\begin{array}{r}
w(x)=\frac{\tau_{\max }}{k_{s}}\left(\frac{e^{\alpha x}-e^{-\alpha x}}{e^{\alpha x_{1}}-e^{-\alpha x_{1}}}\right) \\
\tau(x)=\tau_{\max }\left(\frac{e^{\alpha x}-e^{-\alpha x}}{e^{\alpha x_{1}}-e^{-\alpha x_{1}}}\right)
\end{array}
$$

\subsection{SOLUÇÃO NUMÉRICA}

Para obtenção da solução numérica utilizou-se o Método das Diferenças Finitas (MDF), que se baseia na aproximação de derivadas por diferenças finitas. Com isso, a derivada de segunda ordem da função deslocamento $\mathrm{w}$ em relação à posição x é obtida a partir da Equação 13.

$$
\frac{d^{2} w}{d x^{2}}=\frac{w_{i-1}-2 w_{i}+w_{i+1}}{\Delta x^{2}}
$$

Em que:

$\mathbf{d}^{\mathbf{2}} \mathbf{w} / \mathbf{d x}^{\mathbf{2}}=$ derivada de segunda ordem do deslocamento relativo em relação à posição $\mathrm{x}$;

$\mathbf{w}_{\mathrm{i}-1}=$ deslocamento relativo no nó anterior ao ponto de aplicação da Equação 13;

$\mathbf{w}_{\mathbf{i}}=$ deslocamento relativo no ponto de aplicação da Equação 13;

$\mathbf{w}_{\mathbf{i + 1}}=$ deslocamento relativo no nó seguinte ao ponto de aplicação da Equação 13;

$\Delta \mathbf{x}=\mathrm{Em}$ que $\Delta \mathrm{x}=\mathrm{x}_{1} /(\mathrm{N}-1)$, com $\mathrm{N}$ igual ao número de nós da malha utilizada.

Aplicando-se a Equação 13 na Equação 6 obtém-se a Equação 14.

$$
\frac{w_{i-1}-2 w_{i}+w_{i+1}}{\Delta x^{2}}-\alpha^{2} w_{i}=0 \quad \text { Eq.[14] }
$$

Após desenvolver os termos da Equação 14 chega-se a Equação 15.

$$
-w_{i-1}+r w_{i}-w_{i+1}=0
$$

Em que:

$$
r=2+\alpha^{2} \Delta x^{2}
$$

Considerando um elemento infinitesimal situado na posição $\mathrm{x}_{1}$, como exibido na Figura 4 , ao aplicar as condições de equilíbrio chega-se à Equação 16.

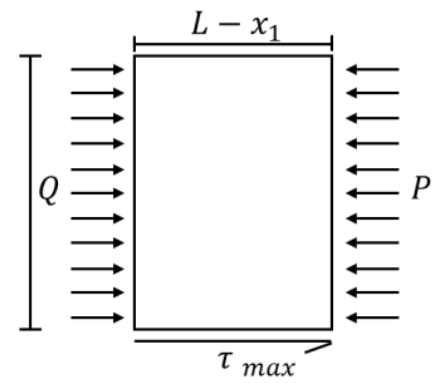

FIGURA 4: Elemento infinitesimal adotado na elaboração do modelo numérico. FONTE: Autoria própria.

$$
Q=P-c\left(L-X_{1}\right)
$$

Em que:

$\mathbf{Q}=$ força horizontal aplicada na posição $\mathrm{x}_{1}$, quando $\mathrm{x}_{1}<\mathrm{L}$.

Sabe-se ainda que no ponto analisado $\left(x_{1}\right)$, a condição de contorno natural é dada pela Equação 17, que substituída na Equação 15, resulta na Equação 18.

$$
\begin{aligned}
{\left[\frac{\mathrm{dw}}{\mathrm{dx}}\right]_{\mathrm{x}_{1}} } & =\frac{\mathrm{Q}}{\mathrm{EH}} \\
-2 \mathrm{w}_{\mathrm{i}-1}+r \mathrm{w}_{\mathrm{i}} & =\frac{2 \Delta \mathrm{xQ}}{\mathrm{EH}}
\end{aligned}
$$

Assim, a solução numérica obtida pelo método das diferenças finitas em função do deslocamento relativo $\mathrm{w}(\mathrm{x})$ pode ser obtida através das matrizes representadas na Equação 19.

$$
\left[\begin{array}{ccccccc}
1 & 0 & 0 & 0 & 0 & \cdots & 0 \\
-1 & r & -1 & 0 & 0 & \cdots & 0 \\
0 & -1 & r & -1 & 0 & \cdots & 0 \\
0 & 0 & -1 & r & -1 & \cdots & 0 \\
0 & 0 & 0 & -1 & \ddots & \ddots & 0 \\
\cdots & \cdots & \cdots & \cdots & \ddots & \ddots & -1 \\
0 & 0 & \cdots & 0 & 0 & -2 & r
\end{array}\right] \cdot\left[\begin{array}{c}
w_{1} \\
w_{2} \\
w_{3} \\
w_{4} \\
\vdots \\
w_{N-1} \\
w_{N}
\end{array}\right]=\left[\begin{array}{c}
0 \\
0 \\
0 \\
0 \\
\vdots \\
0 \\
\frac{2 \Delta x Q}{E H}
\end{array}\right] \text { Eq.[19] }
$$

\subsection{IMPLEMENTAÇÃO COMPUTACIONAL}

$\mathrm{Na}$ implementação computacional, utilizou-se o programa FORTRAN 90. O algoritmo desenvolvido permite a escolha do tipo de resolução: numérica via MDF ou analítica. 
Através da inserção dos dados do modelo físico é possível a impressão dos deslocamentos relativos e tensão cisalhante em função da posição horizontal no interior do bloco. A força total aplicada será dividida pelo valor do número de incrementos de força pretendido pelo usuário. Sendo assim, o programa realizará os cálculos para valores de parcelas menores de força, até que seja atingido o valor da força total. Dessa maneira é possível verificar o comportamento do sistema na medida em que se aumenta a intensidade da força segundo incrementos (ou parcelas) de mesmo valor (igual a F/NINCF). O processo é detalhado no esquema apresentado na Figura 5.
TIPOSOLUCAO é o tipo de solução escolhido pelo usuário (analítica ou numérica), F é força total aplicada, $\mathrm{H}$ é a altura do bloco, $\mathrm{k}_{\mathrm{s}}$ é o modulo de rigidez cisalhante, $L$ é o comprimento do bloco, v é o coeficiente de Poisson, c é a coesão, E é o módulo de elasticidade e NINCX é o número de incrementos de posição, NINCF é o número de incrementos de força. As situações (i) e (ii) são mostradas na Figura 6. Na situação (i) a resistência ao cisalhamento do bloco ainda não foi atingida e é proporcional ao valor do deslocamento relativo. Na situação (ii) a tensão cisalhante atuante atinge o valor da resistência ao cisalhamento e a partir desse momento se torna constante.

(1) Dados de entrada: TIPOSOLUCAO $F, H, k_{s}, L, v, c, E$, NINCX, NINCF

(2) Cálculo de $w_{1}$ (deslocamento relativo máximo)

Os passos a seguir encontram-se dentro do loop de incrementos de força (portanto são repetidos NINCF vezes) até que o valor de força total (F) seja atingido.

(3) Cálculo de $x_{1}$ : Método de Newton Raphson

(4) Avaliar $x_{1}$, determinar as condições de contorno e o tamanho $d x$

(4.1) Se $x_{1}<L$ então fazer: $d x=x_{1} /(N I N C X-1)$

(4.2) Se $x_{1} \geq L$ então fazer: $d x=L /(N I N C X-1)$

(5) Se TIPOSOLUCAO $=1$, então calcular pela solução analítica

(5.1) Se $x_{1}<L$ então:

- Para $0<x<x_{1}$, w é dado pela Equação $11 \mathrm{e}$ $\tau(x)=w(x) \cdot k_{s}$

!(Situação i)

- $\quad \operatorname{Para} x_{1}<x \leq L, w=w_{1}$ e $\tau(x)=w(x) \cdot k_{s}$

!(Situação ii)

(5.2) Se $x_{1} \geq L$ então: $w$ é dado pela Equação 12 e $\tau(x)=w(x) \cdot k_{s} \quad$ !(Situação iii)

(6) Se TIPOSOLUCAO = 2, então calcular pela solução numérica

(6.1) Se $x_{1}<L$ então: a força horizontal aplicada em $x_{1}$ é dada pela equação: ! !(Situação i)

$\mathrm{Q}=\mathrm{F}-\mathrm{c} .\left(\mathrm{L}-\mathrm{x}_{\mathrm{I}}\right)$

(6.2) Se $x_{1} \geq L$ então: a força horizontal utilizada é $Q=F$

!(Situação iii)

(7) Impressão dos resultados

FIGURA 5: Representação esquemática do algorítmico de programação adotado.

FONTE: Autoria própria.

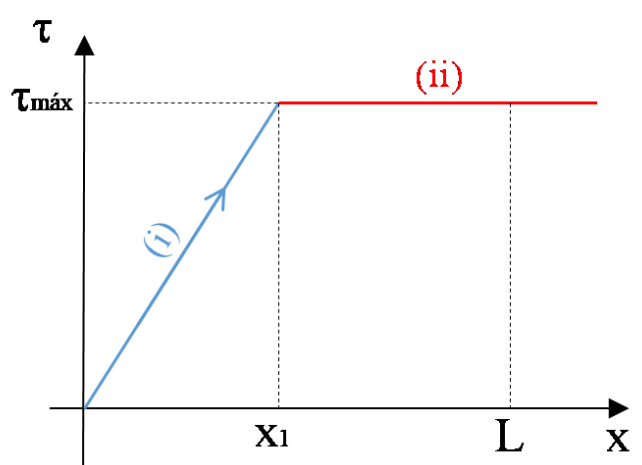

FIGURA 6: Gráfico representativo das situações (i) e (ii).

FONTE: Autoria própria. 
Na Figura 7 está representada a situação (iii) na qual a força atuante não mobiliza toda a resistência ao cisalhamento ao longo do comprimento do bloco.

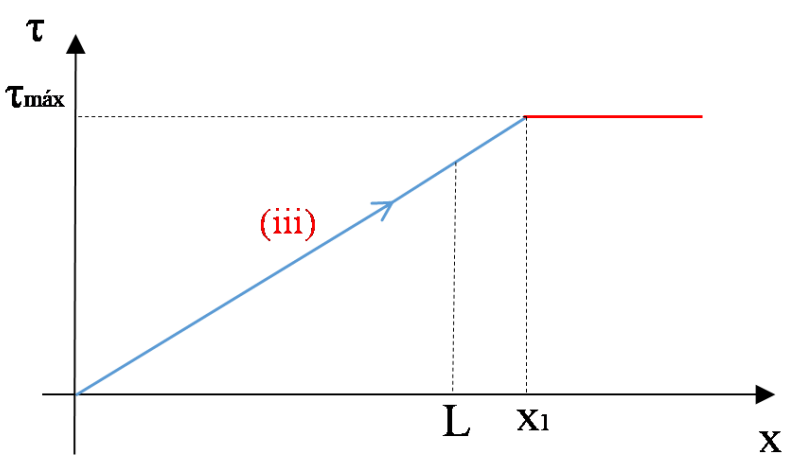

FIGURA 7: Gráfico representativo da situação (iii). FONTE: Autoria própria.

\section{RESULTADOS E DISCUSSÕES}

Afim de se validar a solução via MDF com implementação computacional realizada neste trabalho, análises foram realizadas visando a comparação com a solução analítica proposta por Hird e Russel (1990) e ainda avaliando a influência do coeficiente de cisalhamento, força longitudinal e coeficiente de Poisson na tensão de cisalhamento.

O modelo de meio descontínuo adotado para a validação da implementação computacional e análises está representado na Figura 8. Trata-se de um bloco representativo de um elemento de solo com coeficiente de Poisson (v) igual a 0 , módulo de elasticidade (E) igual a $100000 \mathrm{kN} / \mathrm{m}^{2}$, coesão (c) de $30 \mathrm{kN} / \mathrm{m}^{2}$, altura (H) de 1 metro e comprimento (L) igual a 10 metros, preso na extremidade esquerda e sob atuação de uma força de $400 \mathrm{kN}$ na extremidade direita livre. O bloco situa-se entre duas descontinuidades, sendo a superior lisa e a inferior rugosa com coeficiente de cisalhamento $\left(k_{s}\right)$ igual a $10000 \mathrm{kN} / \mathrm{m}$.

\subsection{SOLUÇÃO NUMÉRICA E SOLUÇÃO ANALÍTICA}

Os gráficos contendo as curvas comparativas entre a solução analítica e numérica com implementação computacional estão apresentados nas Figuras 9 e 10.

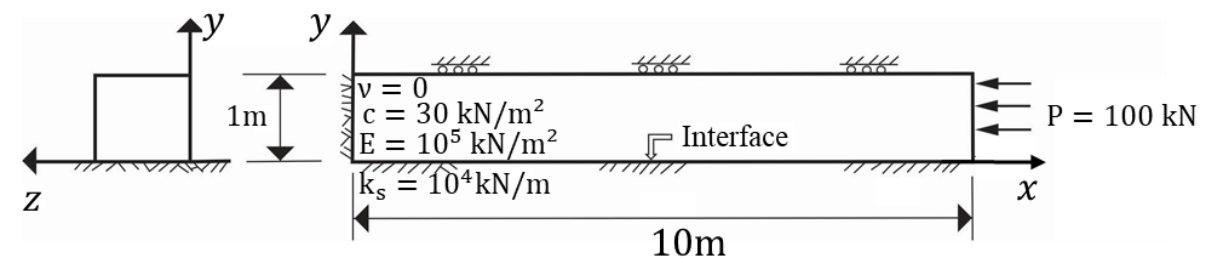

FIGURA 8: Modelo físico adotado para validação das soluções e implementação computacional. FONTE: Autoria própria.



FIGURA 9: Gráfico de tensão versus posição comparando os modelos analítico e numérico. FONTE: Autoria própria. 


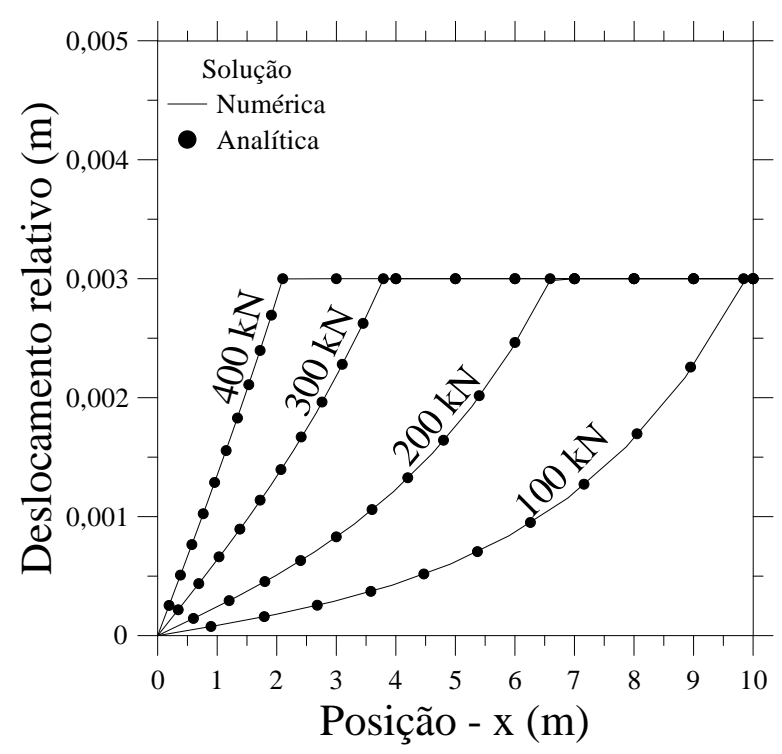

FIGURA 10: Gráfico de deslocamento relativo versus posição comparando os modelos analítico e numérico. FONTE: Autoria própria.

É possível notar a coincidência entre as duas soluções para os quatro níveis de força considerados para essa análise. Esse fato atesta que o modelo numérico utilizado neste trabalho pode ser um bom estimador do comportamento do sistema considerado e ainda valida a implementação computacional e o algoritmo utilizado. Tal fato, era esperado, visto serem as soluções numéricas já apresentadas nos outros trabalhados de referência para esse artigo bastante próximas da analítica.

\subsection{COEFICIENTE DE CISALHAMENTO}

O gráfico da Figura 11 contém as curvas da tensão cisalhante versus a posição para diferentes valores de coeficiente de cisalhamento $k_{s}\left(10^{2}, 10^{4}\right.$ e $10^{6} \mathrm{kN} / \mathrm{m}$ ) considerando-se um carregamento de $400 \mathrm{kN}$. O objetivo dessa análise é validar o modelo proposto por Goodman (1976) dentro da implementação computacional via MDF realizada.

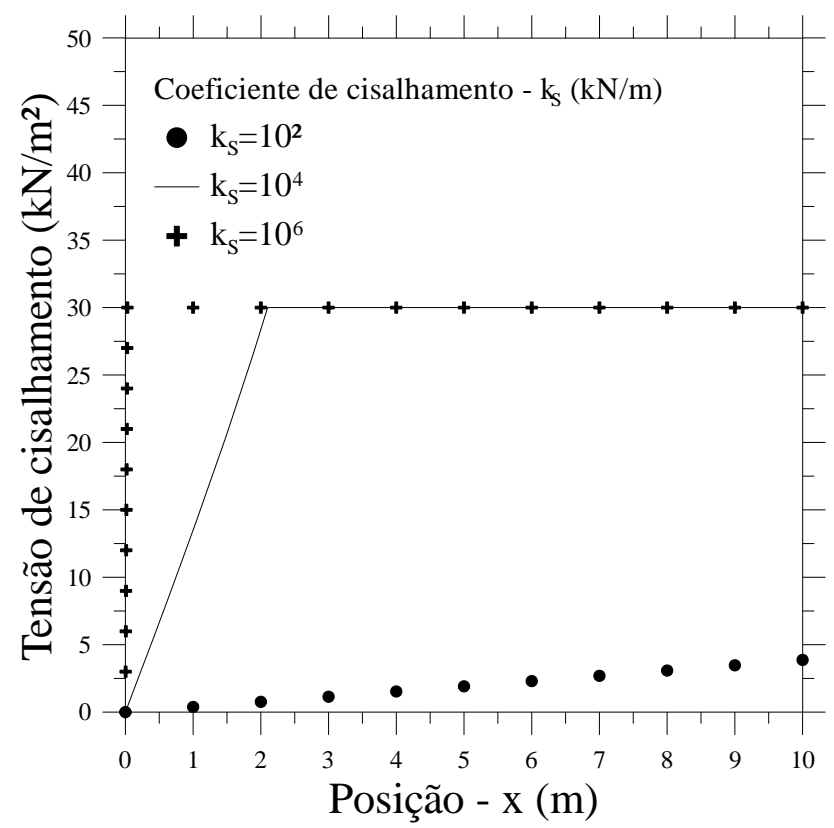

FIGURA 11: Distribuição de tensões para diferentes valores de coeficiente de cisalhamento.

FONTE: Autoria própria. 
A partir da interpretação das curvas da Figura 11, é possível afirmar que a solução numérica proposta neste trabalho está em acordo com o esperado no modelo adotado; quanto maior o coeficiente de cisalhamento maior será a porção do bloco que terá sua coesão mobilizada. A interpretação das curvas da Figura 11, permitem concluir que a solução numérica proposta neste trabalho está em acordo com o esperado no modelo adotado, quanto maior o coeficiente de cisalhamento maior será a porção do bloco que terá sua coesão mobilizada.

\subsection{FORÇA LONGITUDINAL}

O gráfico da Figura 12 ilustra como ocorre a variação de tensão cisalhante no interior do sistema para diferentes níveis de carregamento, mantendo-se os demais dados originais do sistema aqui considerado, considerando-se a solução numérica proposta e implementada computacionalmente. Assim como obtido através da solução analítica como encontrado em Hird e Russel (1990); é possível notar que baixos níveis de carregamento podem não ser suficientes para solicitar toda a coesão do material. Além disso, com o aumento do módulo da força aplicada na extremidade livre, o patamar de tensão (equivalente ao valor de coesão do material) será atingindo em posições cada vez mais próximas da extremidade confinada.

\section{CONCLUSÃO}

As soluções (analítica e numérica) apresentadas permitem prever o deslocamento relativo e a distribuição das tensões de cisalhamento em um maciço rochoso limitado pelas descontinuidades com base no modelo de Goodman (1976).

Os modelos propostos apresentam resultados semelhantes aos obtidos no trabalho realizado por Hird e Russel (1990).

A partir dos dados e dos respectivos gráficos obtidos nas diversas análises realizadas, é possível observar que o modelo numérico apresentou resultados condizentes com aqueles obtidos no modelo analítico, portanto é possível afirmar que modelo numérico proposto neste trabalho pode ser um bom estimador do comportamento do sistema mecânico apresentado.

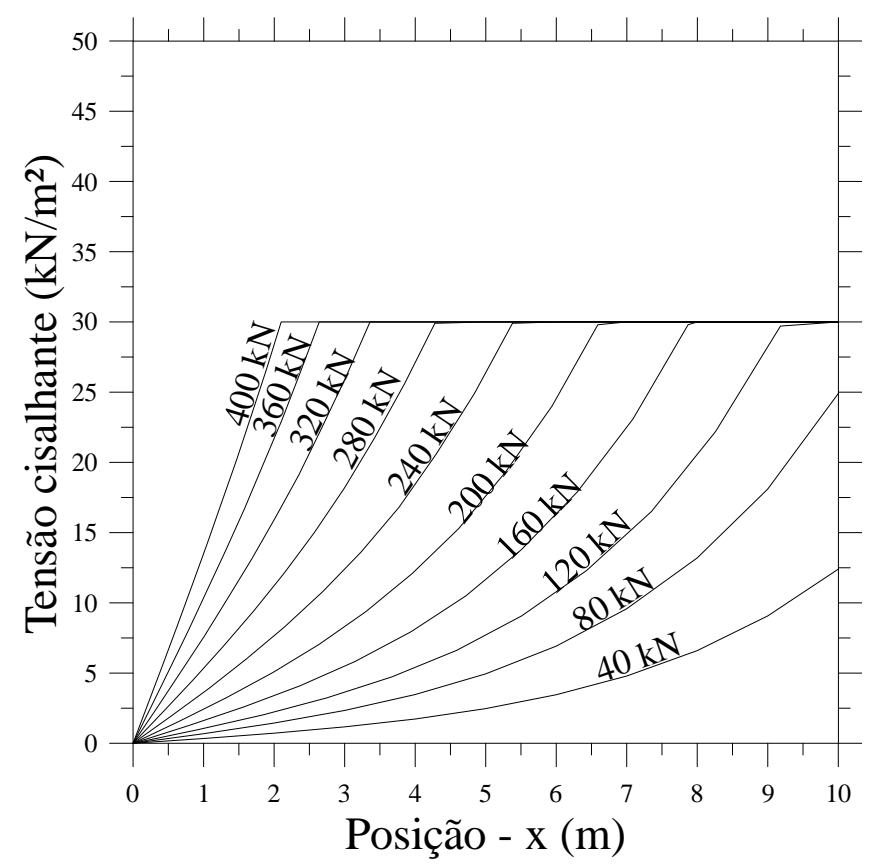

FIGURA 12: Distribuição da tensão cisalhante para diferentes níveis de carregamento. FONTE: Autoria própria. 
Como contribuição deste trabalho para a área de geotecnia cita-se o fato de que o programa executável gerado pode auxiliar no estudo e previsão do comportamento de sistemas mecânicos semelhantes ao aqui proposto. Nesse sentido, o usuário do programa tem a possibilidade de, conforme sua necessidade, variar os valores de: número de pontos nodais, força aplicada, altura e comprimento do bloco, coeficiente de Poisson, coesão, módulo de elasticidade e coeficiente de cisalhamento do plano da descontinuidade.

Além disso, outra contribuição para a área de geotecnia, é o fato de que o presente estudo também auxilia no desenvolvimento de novas metodologias de análise de estabilidade de taludes e escavações subterrâneas que objetivem resultados mais representativos da realidade do que aqueles obtidos atualmente ao se considerar o meio como contínuo e utilizando parâmetros geotécnicos equivalentes.

Por fim, considera-se como sugestão a análise de outros modelos que não o de Goodman (1976) ou ainda a utilização de outros métodos numéricos como por exemplo o Métodos dos Elementos Finitos Estendidos para analisar o comportamento mecânico de maciços rochosos contendo descontinuidades.

\section{AGRADECIMENTOS}

Ao engenheiro de minas Ronald Dantas pelo auxílio nas atividades de programação. À CAPES e a FAPEMIG pelo apoio financeiro a realização dessa pesquisa.

\section{REFERÊNCIAS BIBLIOGRÁFICAS:}

BANDIS, S.C., Lumsden, A.C., Barton, N. Fundamentals of rock joint deformation. International Journal of Rock Mechanics and Mining Science and Geomechanics. 1983, 249-268 p.

GOODMAN, R.E. Methods of Geological Engineering in Discontinuous Rocks. West publishing Co., New York, 1976.
GRASSELLI, G. Shear strength of rock joints based on quantified surface description. Rock Mechanics and Rock Engineering 39 (4), 2006, 295-314.

HIRD, C. C.; Russell, D. A benchmark for soil-structure interface elements. Computers and Geotechnics, v. 10, n. 2, 1990, pp. 139-147.

LADANYI, B., Archambault, G. Simulation of the shear behavior of a jointed rock mass. 11th Symposium on Rock Mechanics, Berkeley, 1969, pp. 105-125.

NIKOLIĆ, Mijo; ROJE-BONACCl, Tanja; IBRAHIMBEGOVIĆ, Adnan. Overview of the numerical methods for the modelling of rock mechanics problems. Tehnički vjesnik, v. 23, n. 2, p. 627, 2016.

PATTON, F.D. Multiple modes of shear failure in rock. Proc. 1st Cong. ISRM, Lisbon, vol. 1, 1966, pp. 509-513.

PEREIRA, Anderson Resende. Modelagem numérica não linear física via MEF de estruturas de solos reforçados. 2003.

PLESHA, M.E. Constitutive models for rock discontinuities with dilatancy and surface degradation. International Journal of Rock Mechanics and Science and Geomechanics. 1988.

SAEB, S., Amadei, B. Modeling rock joints under shear and normal loading. International Journal of Rock Mechanics and Science and Geomechanics Abstracts 29, 1992, 267-278.

SEIDEL, J.P., Haberfield, C.M. Laboratory testing of concrete-rock joints in constant normal stiffness direct shear. Geotechnical Testing Journal 25 (4). 2002a.

SEIDEL, J.P., Haberfield, C.M. A theoretical model for rock joints subjected to constant normal stiffness direct shear. International Journal of Rock Mechanics and Mining Science, 2002b, 539-553.

USEFZADEH, Arash et al. Empirical and mathematical formulation of the shear behavior of rock joints. Engineering Geology, v. 164, p. 243-252, 2013.

VARGAS, Jr. E. A. Modelagem numérica de maciços rochosos fraturados aplicada ao problema de erosão a jusante de vertedouros de grandes barragens. XIX Seminário Nacional de Grandes Barragens. (SNXIX), 1991, pp. 283-292. 